\title{
Correlação entre capacidade funcional e capacidade pulmonar em pacientes com doença pulmonar obstrutiva crônica
}

\section{Correlation between functional capacity and lung capacity in patients with Chronic Obstrutive Pulmonary Disease}

\author{
Karla Katarine Rodrigues Teixeira Bastos ${ }^{1}$ (D) , Roseane Chaves de Araújo Oliveira ${ }^{2}$ (D) , Winnie Alves Moreira Lima ${ }^{2}$ (D) , \\ Ricardo Ribeiro Badaró ${ }^{1}$ (D), Keyla Iane Donato Brito Costa $^{1}$ (D), Pablo Luiz Santos Couto ${ }^{1}$ (])
}

1. Docente do curso de Fisioterapia pela Faculdade Guanambi (FG), Guanambi, BA, Brasil. 2. Discente do curso de Fisioterapia pela Faculdade Guanambi (FG), Guanambi, BA, Brasil.

\section{Resumo}

Introdução: o a avaliação da capacidade pulmonar é realizada por meio da espirometria, que permite avaliar os principais parâmetros respiratórios. Entre as formas de avaliar a capacidade funcional, tem-se o teste de caminhada de seis minutos que proporciona uma análise global dos sistemas. Essas formas de análise funcional contribuem tanto para a compreensão do impacto da doença e do seu estadiamento clínico, como para a elaboração de prognóstico. Objetivo: correlacionar a capacidade funcional e a capacidade pulmonar em pacientes com DPOC. Métodos: tratou-se de uma pesquisa transversal, quantitativa, na qual foi aplicada a espirometria e o TC6m em pacientes atendidos pelo Programa Respira Bahia, inserido na Polimeg de Guanambi, entre os meses de abril a novembro de 2016. Resultados: observou-se correlação moderada positiva da variável VEF1 em relação ao TC6m. Conclusão: concluise que, nos pacientes com DPOC analisados, o comprometimento da função pulmonar está diretamente relacionado com a piora da capacidade funcional.

Palavras-chave: Doença Pulmonar Obstrutiva Crônica. Espirometria. Teste de caminhada de seis minutos

\begin{abstract}
Introduction: The assessment of pulmonary capacity is performed through spirometry, which allows the evaluation of the main respiratory parameters Among the ways of assessing functional capacity, we have the 6-minute walk test that provides an overall analysis of the systems. These forms of functional analysis contribute both to understanding the impact of the disease and its clinical staging, as well as to the prognosis. Objective: To correlate functional capacity and lung capacity in patients with COPD. Methods: This was a cross-sectional, quantitative study in which spirometry and 6MWT were performed in patients attended by the "Respira Bahia" Program, inserted in the Polimeg de Guanambi, between April and November 2016. Results: positive correlation of the FEV1 variable in relation to the 6MWT. Conclusion: it was concluded that, in patients with COPD, pulmonary function impairment is directly related to worsening of functional capacity.
\end{abstract}

Key words: Chronic Obstrutive Pulmonary Disease. Spirometry. six-minute Walk Test

INTRODUÇÃO

A doença pulmonar obstrutiva crônica (DPOC) é uma doença respiratória crônica em resposta a uma inflamação anormal dos pulmões provocada por várias substâncias inaladas, sendo causada primariamente pelo tabagismo". A DPOC "é um importante problema de saúde e estima-se que será a quinta principal causa de incapacidade e a terceira principal causa de mortalidade no mundo até $2020^{\prime \prime 2}$. A DPOC é a responsável por 3 milhões de mortes a cada ano ${ }^{3}$.

A DPOC provoca uma deterioração progressiva da função respiratória, bem como da musculatura periférica ao longo do tempo, com manifestações sistêmicas que comprometem a capacidade funcional, sendo evidenciadas pela fadigabilidade e/ou dispneia, causando impacto negativo na qualidade de vida desses pacientes, tornando-os dependentes ou incapacitados para realizar suas atividades de vida diária (AVD's) ${ }^{4}$.
Devido a essas alterações, a avaliação da capacidade funcional de indivíduos com DPOC tornou-se alvo de muitos estudos. Para a avaliação da capacidade funcional, utilizamse, frequentemente, testes físicos funcionais, os quais são aplicados com o intuito de complementar a avaliação estática de indivíduos com DPOC, monitorar a efetividade do tratamento e estabelecer o prognóstico desses indivíduos.

Entre os meios utilizados para avaliar a capacidade funcional, tem-se o teste de caminhada de seis minutos (TC6m) que proporciona uma análise global dos sistemas respiratório, cardíaco e metabólico ${ }^{5}$; já a espirometria é recomendada como forma de triagem para todos os indivíduos com sintomas e/ou exposição de risco à DPOC .

Estudos realizados ${ }^{7}$ mostram que, na avaliação das 
enfermidades pulmonares crônicas somadas aos testes estáticos (espirometria), os testes dinâmicos (teste submáximo - TC6m) são cada vez mais prevalentes. Essas formas de análise funcional, segundo esses autores, contribuem tanto para a compreensão do impacto da doença e do seu estadiamento clínico, como para a elaboração de prognóstico ${ }^{11}$.

Apesar de haver quantidades significativas de estudos sobre a capacidade pulmonar e a capacidade funcional em pacientes com doença pulmonar obstrutiva crônica, estes abordam o tema de forma isolada, o que pode requerer um maior tempo para a confirmação do diagnóstico e a elaboração de um tratamento mais adequado para cada paciente. Sendo assim, avaliar simultaneamente essas capacidades é de suma importância para a elaboração de estratégias que possibilite um tratamento melhor e mais completo, que proporcione maior custo-benefício para os pacientes e para o sistema de saúde pública, se tornará mais seguro e fidedigno.

Dessa forma, o objetivo do presente artigo é correlacionar a capacidade funcional e a capacidade pulmonar em pacientes com doença pulmonar obstrutiva crônica.

\section{MÉTOdOS}

Trata-se de um estudo transversal, quantitativo, que foi aprovado pelo Comitê de Ética da FAINOR sob protocolo número 54338816.5.0000.5578. Todos os pacientes que participaram do estudo assinaram o Termo de Consentimento Livre e Esclarecido de acordo com a resolução CNS no. 466/2012. O estudo foi realizado na Policlínica Médica de Guanambi POLIMEG, em que está inserido o Programa Respira Bahia que é o único programa local de assistência em saúde para pacientes portadores de Asma e DPOC. A coleta de dados foi realizada no período de abril a novembro de 2016, duas vezes por semana, em dias destinados ao atendimento da médica pneumologista do programa.

Inicialmente, realizou-se uma busca nos prontuários, obtendo uma população de 66 pacientes atendidos pelo Programa Respira Bahia, com diagnóstico de DPOC, com idade superior a 18 anos, de ambos os sexos. Posteriormente, foi realizada uma breve entrevista com os pacientes para obtenção de informações pertinentes à pesquisa, tais como tempo de diagnóstico, tempo de exposição ao tabagismo e outros fatores de risco. Foram excluídos pacientes que apresentavam limitações ortopédicas e cognitivas, angina instável, infarto agudo do miocárdio recente, pressão arterial (PA) em repouso acima de $180 \times 100 \mathrm{mmHg}$, arritmia cardíaca não controlada, frequência cardíaca (FC) em repouso acima de 120 bpm, saturação periférica da oxigenoglobina $\left(\mathrm{SpO}_{2}\right)$ basal $<90 \%$ em repouso e pacientes que apresentaram valores acima de 4 na escala de Borg8. Essa escala é utilizada para graduar o nível de dispneia, e é quantificada de 0 a 10, sendo que 0 representa nenhum sintoma e 10 representa sintoma máximo. Foram pré-selecionados 18 pacientes que se enquadraram dentro desses critérios. Destes, 4 foram eliminados após realização da espirometria, pois apresentaram distúrbio misto (restritivo e obstrutivo).

Foram avaliados antes da realização dos testes os sinais vitais: $\mathrm{PA}, \mathrm{FC}, \mathrm{SpO}_{2}$, frequência respiratória (FR) e ausculta pulmonar (AP), para verificar se os pacientes estavam aptos para realizar os testes e coletados dados como idade, sexo, altura, peso e distância prevista para o teste de caminhada. Os testes foram divididos em duas etapas: a primeira etapa foi a realizada por meio do exame de espirometria, utilizando para o teste o aparelho espirômetro da marca $\mathrm{Koko}^{\circledR}$ e a segunda etapa foi realizada por meio do teste de caminhada de 6 minutos.

O teste espirométrico foi realizado conforme estudo com adultos saudáveis em uma província do Irã, o qual relata que o indivíduo deve estar sentado confortavelmente com a cabeça neutra e os pés apoiados no chão9. Foi colocado um clipe nasal para não haver escape de ar, solicitada uma inspiração profunda até a capacidade pulmonar total (CPT) e, em seguida, após acoplar a boca no bocal, uma expiração de forma rápida e contínua. As medidas espirométricas foram mensuradas antes e 15 minutos após o uso do broncodilatador salbultamol (400ug). O procedimento foi repetido até o indivíduo alcançar três curvas aceitáveis pelo espirômetro, sem exceder um máximo de oito tentativas. Foi selecionada para análise a melhor das três curvas. Foi analisado o volume expiratório forçado no primeiro segundo (VEF1) em litros e em percentual do previsto (VEF1\%) pós-broncodilatador. Após a realização da espirometria, a médica pneumologista responsável pelo programa laudou os exames classificando os padrões segundo sua gravidade: em leve, moderado e grave conforme preconizado pela Global Initiative for Chronic obstructive Pulmonary Disease (GOLD) ${ }^{14}$.

O próprio equipamento emitiu a interpretação da espirometria, informando se a função pulmonar encontrava-se normal ou se havia presença de padrão obstrutivo, restivo ou misto, classificando esses padrões segundo sua gravidade: em leve, moderado e grave e os eles foram confrontados com os padrões emitidos pela Global Initiative for Chronic obstructive Pulmonary Disease (GOLD) ${ }^{10}$. Posteriormente, foram laudados pela médica responsável pelo programa.

O TC6m foi aplicado conforme preconizado pela American Thoracic Society (ATS) (2002a), a qual recomenda que seja "em um corredor plano de 30m, demarcado a cada metro". Os pacientes foram orientados a caminhar a maior distância possível e receberam incentivo verbal padronizado ${ }^{11}$. A FC, a $\mathrm{SpO}_{2}$ e o índice de dispneia foram mensurados a cada minuto. Antes e após a realização de cada teste, para melhor fidedignidade dos resultados e para prevenir qualquer intercorrência aos pacientes, $\mathrm{PA}, \mathrm{FC}, \mathrm{FR}$, e $\mathrm{SpO}_{2}$ foram mensurados, além da ausculta cardíaca e respiratória e a avaliação do nível de dispneia. Foram realizados dois testes, com intervalo de 30 minutos entre eles. Considerou-se para análise a distância percorrida do segundo teste e o valor previsto para o teste ${ }^{12}$. Esse valor previsto foi 
encontrado por meio da fórmula proposta por Enright e Sherrill, para homens: distância TC6m $=(7,57 \times$ altura $\mathrm{cm})-(5,02 \times$ idade) $-(1,76$ x peso $\mathrm{kg})-309$; e para mulheres: distância TC6m $=(2,11 \times$ altura $\mathrm{cm})-(2,29 \times$ peso $\mathrm{kg})-(5,78 \times$ idade $)+667^{13}$.

Os dados obtidos foram tabulados e analisados no software SPSS versão 21 . A estatística descritiva foi realizada por meio de medidas de tendência central, desvio padrão, frequências e valor máximo e mínimo. Posteriormente, foi realizada a prova de normalidade de Shapiro Wilk para as variáveis DTC6m e VEF1, que apresentaram distribuição anormal; portanto, a análise de correlação entre essas variáveis foi realizada por meio do coeficiente de Spearman. Para a interpretação dos resultados, assumiu-se o nível de significância de 95\%.

\section{RESULTADOS}

Foram selecionados 18 pacientes, sendo que 4 foram excluídos após apresentar distúrbio misto na espirometria, totalizando uma amostra de 14 pacientes, sendo $71 \%$ do sexo masculino e $29 \%$ do sexo feminino com média de idade $74 \pm 8.9$ anos. Com relação ao tabagismo, dez (71\%) dos pacientes são ex-tabagistas, três $(22 \%)$ são tabagistas ativos e um (7\%) foi tabagista passivo. Desses 14 pacientes, $14 \%$ tiveram contato com fumaça de fogão à lenha. Dentro dessa população estudada, $29 \%$ dos pacientes foram diagnosticados com DPOC há mais de 10 anos, e $71 \%$ tiveram o diagnóstico há menos de 10 anos. Dessa amostra, 35\% dos pacientes pararam de fumar no mesmo ano do diagnóstico e $43 \%$ fumaram por um período superior a 50 anos.

Ao analisar o TC6m, pode-se observar que a média da $\mathrm{SpO2,}$ FC e da PA diastólica tanto pré quanto pós-teste mantiveramse dentro dos parâmetros de normalidade, apesar da gravidade da doença. Quando analisada a PA sistólica pré e pós-teste, observou-se um aumento significativo, e a FR apresentou um aumento no pós-teste. Porém esses sinais que descompensaram não apresentaram riscos aos pacientes por terem sido normalizados em poucos minutos. Sobre a escala de Borg, $36 \%$ dos pacientes mantiveram seus valores estáveis durante a realização do teste, e $64 \%$ tiveram uma variação gradativa dos valores ao decorrer do percurso.

Ao analisar o VEF1 da população estudada, observa-se um valor de 1,27 atingido na melhor das três curvas que corresponde a $44 \%$ sobre o valor de referência (figura 1 ).

Baseado no que preconiza a ATS, foram realizados dois TC6m com intervalo de 30 minutos entre eles, e selecionado para análise o segundo teste realizado ${ }^{10}$.

Sendo assim, a distância média prevista para os pacientes percorrerem seria de $474 \pm 62.4 \mathrm{~m}$. Na realização do primeiro teste, a média de distância percorrida foi de $368 \pm 162.4 \mathrm{~m}$ e, no segundo teste, obteve-se a média de $407 \pm 168.9 m$ (figura 2).

Ao final do TC6m, percebeu-se que existiram aqueles pacientes (22\%) que caminharam por menos de três minutos, apresentando um nível acentuado de dispneia e exaustão física, $43 \%$ dos pacientes completaram o teste com um valor abaixo do previsto e $35 \%$ terminaram o teste alcançando o limite maior que o previsto, não apresentando alterações físicas e respiratórias aparentes ao final do teste. Isso se deve ao fato de que a fórmula utilizada, refere-se a indivíduos saudáveis, realizando o TC6m sem o incentivo verbal, porém, segundo a ATS, esse teste deve ser realizado com encorajamento verba ${ }^{110,12}$.

Figura 1. Valores da média da espirometria VEF1 pósbroncodilatador em pacientes com DPOC

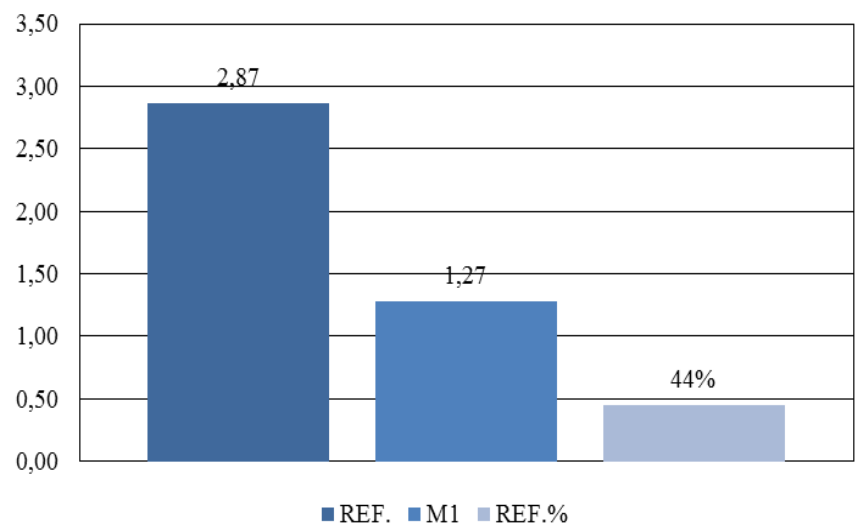

VEF1: volume expiratório forçado no primeiro minuto; Ref: referência; M1: melhor das três curvas; Ref \%: percentual realizado sob a referência.

Figura 2. Média de distância prevista e percorrida pelos pacientes com DPOC no TC6m

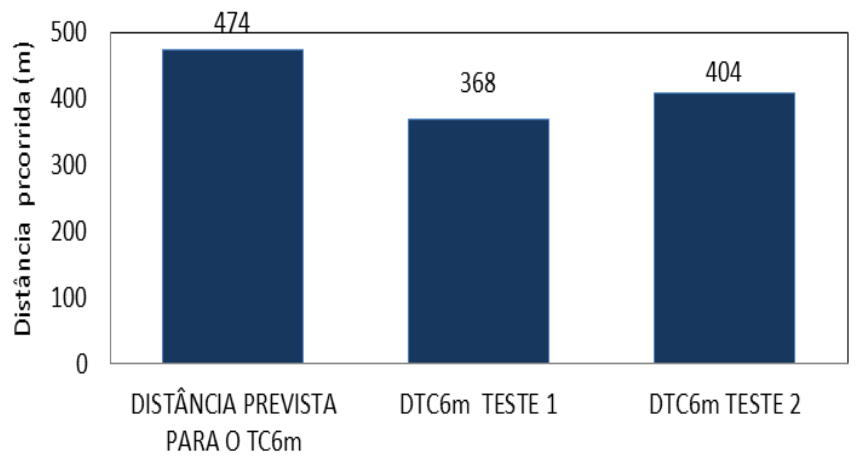

Perfil da capacidade funcional

TC6m: teste de caminhada de seis minutos; DTC6m: distância prevista para o teste de caminhada de 6 minutos.

Ao analisar a correlação entre as variáveis de VEF1 e TC6m, ou seja, capacidade pulmonar e capacidade funcional, foi encontrada uma correlação moderada positiva $(p=0,54)$ (figura 3).

Correlacionado o parâmetro espirométrico VEF1 e a DTC6, por meio do coeficiente de Spearman obteve-se $p=0,54$; pode-se a afirmar que, quanto maior o grau de comprometimento da capacidade pulmonar, maior será sua influência na capacidade funcional do indivíduo. 
Ao analisar separadamente os resultados de acordo com o nível de gravidade da doença, correlacionando-os com a capacidade funcional dos pacientes com DPOC, observou-se que aqueles pacientes com nível de obstrução grave, o que corresponde a $50 \%$ da amostra, apresentaram uma correlação positiva forte $(p=0,77)$. Os pacientes, com nível de obstrução moderada, apresentaram uma correlação positiva moderada $(p=0,54)$ e os pacientes, com nível de obstrução leve, apresentaram uma correlação positiva fraca $(p=0,38)$ (figura 4$)$.

Figura 3. características gráficas da análise de correlação por meio do coeficiente de Spearman do teste de caminhada de seis minutos e da variável espirométrica de pacientes com DPOC

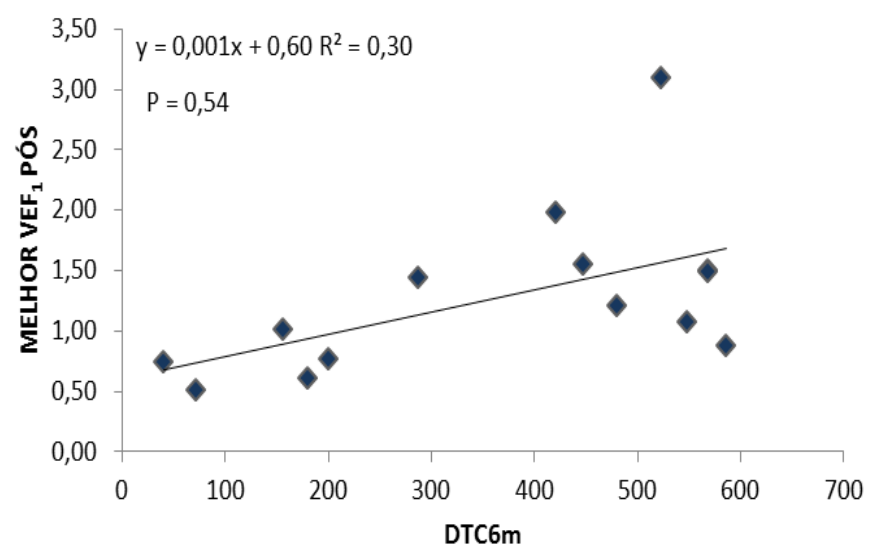

Figura 4. características gráficas da análise de correlação por meio do coeficiente de Spearman do teste de caminhada de seis minutos e da variável espirométrica de acordo o nível de gravidade da DPOC
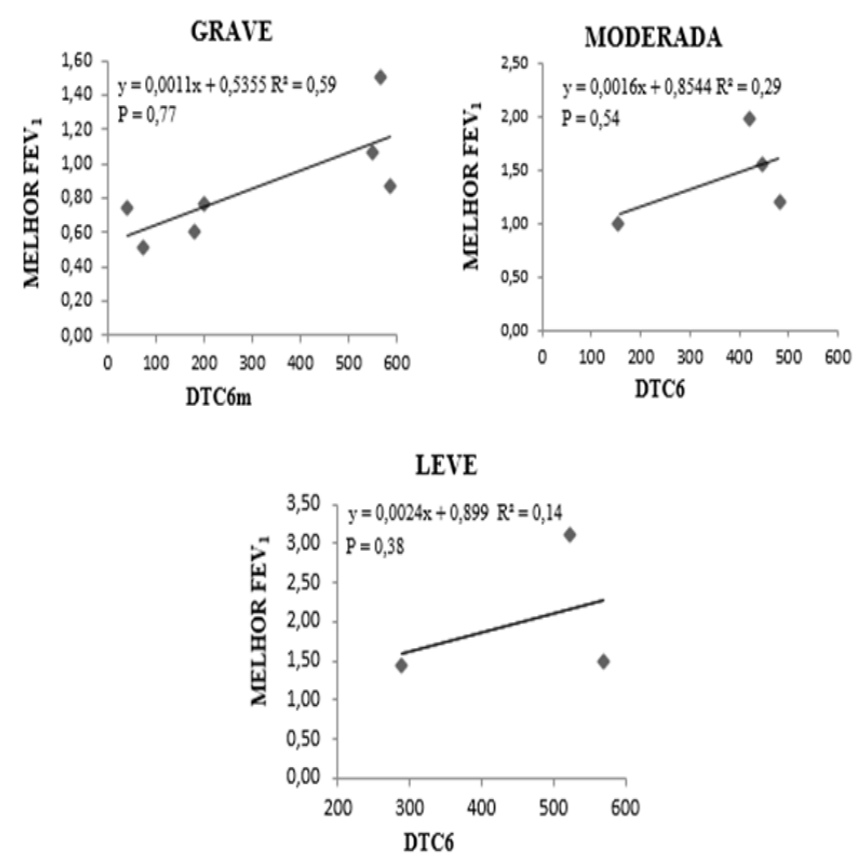

TC6m: teste de caminhada de seis minutos; VEF1: volume expiratório forçado no primeiro minuto; DTC6m: distância prevista para o teste de caminhada de 6 minutos.
Esses resultados deixam explícita a afirmação de que quanto maior for o comprometimento da função pulmonar mais prejudicada estará a capacidade funcional do indivíduo com DPOC grave. Aqueles pacientes que tiveram uma correlação positiva forte apresentaram um comprometimento grave tanto na capacidade pulmonar, quanto na capacidade funcional, o que se pode concluir que essas variáveis são diretamente proporcionais $\left(r^{2}=0,59\right)$. Naqueles pacientes que tiveram uma correlação positiva fraca, apesar de existir correlação entre capacidade pulmonar e capacidade funcional, não se pode afirmar que as variáveis são diretamente proporcionais e que tenha um comprometimento leve tanto da capacidade pulmonar quanto da capacidade funcional devido à variável de determinação neste estudo ser de $r^{2}=0,14$.

\section{DISCUSSÃO}

As características da população deste estudo, bem como a avaliação da gravidade da DPOC, foram analisadas tendo como base a graduação da obstrução conforme preconizado pela GOLD $^{10}$.

A avaliação do nível de gravidade da DPOC nesses pacientes foi analisada, tendo como base a medida do $\operatorname{VEF}_{1}$ pós o uso do broncodilatador, sendo estabelecida a graduação da obstrução de acordo com a preconizada pela GOLD ${ }^{14}$, uma vez que, "a espirometria é um teste funcional amplamente utilizado, e seus parâmetros, sobretudo o $\mathrm{VEF}_{1}$, apresentam boa reprodutibilidade"2-14.

O diagnóstico da DPOC é baseado em avaliação da função pulmonar dos pacientes, dos sintomas, do histórico de exacerbações e da avaliação do fenótipo, porém a exigência fisiopatológica para diagnóstico do DPOC é a presença de limitação ao fluxo aéreo expiratório pós-prova broncodilatadora com redução do VEF1/capacidade vital abaixo dos níveis considerados normais o que indica limitação persistente do fluxo aéreo fator mais importante ao se analisar a função pulmonar dos indivíduos com DPOC 6 .

Na respiração de indivíduos saudáveis, a inspiração se inicia após uma expiração completa, quando o nível respiratório em repouso e a pressão do sistema respiratório chegam a zero. Em pacientes com DPOC, o nível respiratório de repouso pode continuar acima do nível normal por diversos ciclos respiratórios devido ao aprisionamento aéreo. Nos pacientes com doença obstrutiva, o fluxo expiratório encontra-se limitado na respiração espontânea, ocorrendo o início da inspiração antes que a expiração anterior se finalize, fazendo que a diferença de pressão no sistema não chegue a zero, levando a chamada pressão positiva intrínseca ao final da expiração (PEEPi). Por conseguinte, utilizou-se o $\mathrm{VEF}_{1}$ como parâmetro para análise de correlação.

De acordo com o estudo que elaborou uma proposta de padronização do teste de caminhada, a distância percorrida no segundo teste foi maior que a do primeiro, devido ao efeito 
de aprendizado. Por se tratar de um teste que depende da cooperação do paciente, o primeiro teste é utilizado como forma de adaptação do percurso ${ }^{15}$.

Sendo assim, este trabalho vai ao encontro do estudo sobre a correlação entre as provas funcionais respiratórias e o teste de caminhada, em que é afirmado que o estímulo verbal adotado pelos examinadores pode ser o responsável pela melhor performance física apresentada pelos pacientes 16 , ou seja, "durante o TC6m, a estimulação verbal determina melhor rendimento físico em pacientes portadores de enfermidades cardiopulmonares" 3 -17.

O resultado da correlação do parâmetro espirométrico foi encontrado em um estudo realizado com pacientes com DPOC em que, a partir do teste, verificou-se o grau de obstrução do fluxo aéreo ${ }^{18}$, e vai ao encontro da pesquisa realizada com pacientes com a capacidade funcional diminuída, em decorrência da DPOC, que demonstrou que há correlação de variáveis da função pulmonar, como o $\mathrm{VEF}_{1}$, com o trabalho desenvolvido no TC6 $\mathrm{min}^{19}$.

A maior parte dos pacientes eram trabalhadores rurais e possuíam um estilo de vida ativo, e, em decorrência da doença, relataram ter diminuído ou abandonado a realização de atividades de seu cotidiano em consequência da sintomatologia da DPOC, tendo como principais fatores: a fadiga e a dispneia. A atividade física é, muitas vezes, difícil ou impossível para pacientes com DPOC, principalmente para aqueles que apresentam comorbidades comumente encontradas tais como artroses, doenças cerebrovasculares e obesidade, fatores que podem limitar sua capacidade de realizar exercícios, tornando-o sedentário ${ }^{20}$.

Essas alterações afetam a execução das AVD's. Dessa forma, pode-se compreender que a fadiga muscular ocasionada pela DPOC pode ter como um fator desencadeante a hipoxemia arterial, uma vez que esses pacientes necessitam de um maior nível de energia para os músculos respiratórios devido ao aumento da ventilação. Em decorrência disso, esses pacientes têm uma tendência a reduzir cada vez mais suas atividades, o que leva a um comprometimento maior da doença, conduzindo a uma maior intensidade dos sintomas e menores níveis de atividade.

A correlação moderada positiva da população total foi obtida mesmo com os pacientes apresentando a capacidade pulmonar prejudicada, e mostrando uma boa reprodutibilidade no TC6m. No entanto, mesmo seguindo um critério metodológico, alguns aspectos subjetivos podem ter interferido no resultado da análise, tais como, o tempo de exposição à fumaça do fogão à lenha, tempo de inalação da fumaça do cigarro, fumo passivo, sexo, idade, situação socioeconômica, gravidade da doença, tolerância ao exercício, dispneia e exacerbações.

Neste estudo, a capacidade pulmonar correlacionou-se positivamente com a capacidade funcional $(p=0,54)$, indicando que a aplicação do TC6m pode contribuir para a avaliação da deterioração da capacidade pulmonar, além da sobrevida e do nível de funcionalidade dos pacientes com DPOC. O TC6m foi considerado melhor preditor de sobrevida que os parâmetros tradicionais. O estudo ainda afirma que os pacientes com DPOC "que apresentam maior distância percorrida no TC6m possuem maior sobrevida, em relação aos que têm pior rendimento neste teste"21.

\section{CONCLUSÃO}

As análises realizadas permitem concluir que, nos pacientes estudados, o comprometimento na capacidade pulmonar correlaciona-se com a redução da capacidade funcional; entretanto, os resultados apresentados devem ser analisados com cautela, pois a investigação apresenta limitações relacionadas ao tamanho amostral e ao cenário da pesquisa, que impossibilitam a generalização dos resultados para outros pacientes com doença pulmonar obstrutiva crônica.

Este estudo serve como subsídio para a condução de novas investigações na temática, que se fazem necessárias. Sugerese a realização de investigações com amostras maiores e que utilizem outros instrumentos para a medida de capacidade funcional nos pacientes com DPOC.

\section{REFERÊNCIAS}

1. Sociedade Brasileira de Pneumologia e Tisiologia. II Consenso Brasileiro sobre Doença Pulmonar Obstrutiva Crônica - DPOC. "Caracterização da Doença Pulmonar Obstrutiva Crônica (DPOC) - Definição, Epidemiologia, Diagnóstico e Estadiamento". J Bras Pneumol [Internet]. 2004 Nov [acesso 2016 Nov 15];30(supl.5):S1-S5. Disponível em: http://www.jornaldepneumologia.com. br/PDF/Suple_124_40_DPOC_COMPLETO_FINALimpresso.pdf.

2. Torres-Sánchez I, Rodríguez-Alzueta E, Crabera-Martos I, López-Torres I, Moreno-Ramírez MP, Valenza MC. Comprometimento cognitivo em pacientes com DPOC: uma revisão sistemática J Bras Pneumol [Internet]. 2015 Mar-Apr [acesso 2016 Nov 15];4(2):182-190. Disponível em: http://www.scielo.br/pdf/ jbpneu/v41n2/pt_1806-3713-jbpneu-2015000004424.pdf. doi: http://dx.doi. org/10.1590/S1806-37132015000004424.

3. Rabahi MF. Epidemiologia da DPOC: Enfrentando Desafios. Rev Pulmão RJ [Internet]. 2013 Apr [acesso 2016 Nov 15];22(2):4-8. Disponível em: http:// sopterj.com.br/profissionais/_revista/2013/n_02/02.pdf.

4. Santos K, Karloh M, Araújo CLP, D’Aquino AB, Mayer AF. Relationship between the functional status constructs and quality of life in COPD. Rev Fisioter Mov [Internet]. 2014 Jul-Sept [acesso 2016 Nov 15];27(3):361-369. Disponível em: http://www.scielo.br/pdf/fm/v27n3/0103-5150-fm-27-03-0361.pdf. doi: http://dx.doi.org.10.1590/0103-5150.027.003.AO06.

5. Fernandes PM, Pereira NH, Santos ACBC, Soares MESM. Teste de Caminhada de Seis Minutos: avaliação da capacidade funcional de indivíduos sedentários. Rev Bra Cardiol [Internet]. 2012 May-Jun [acesso 2016 Nov 15];25(3):185-191. Disponível em: http://www.rbconline.org.br/wp-content/Archives/v25n3/ v25n03a03.pdf.

6. Koblizek V, Novotna B, Zbozinkova Z, Hejduk K. Diagnosing COPD: advances in training and practice - a systematic review. Adv Med Educ Pract. [Internet]. 
2016 Apr 4; 7:219-31. Disponível em: https://www.ncbi.nlm.nih.gov/pmc/ articles/PMC4825818/pdf/amep-7-219.pdfb. doi: 10.2147/AMEP.S76976.

7. Rodrigues SL, Melo e Silva CA, Lima T, Viegas CAA, Rodrigues MP, Ribeiro FA. Influência da função pulmonar e da força muscular na capacidade funcional de portadores de doença pulmonar obstrutiva crônica. Rev Port Pneumol [Internet]. 2009 Mar [acesso 2016 Nov 15]; 15(2): 199-214. Disponível em: http://www.scielo.mec.pt/pdf/pne/v15n2/v15n2a05.pdf.

8. Jardim JRB, Oliveira JCA, Rufino R. Projeto Diretrizes. In: Sociedade Brasileira de Pneumologia e Tisiologia. Doença Pulmonar Obstrutiva Crônica. São Paulo: AMB; 2001. p. 1-8. Disponível em: http://www.portalmedico.org.br/diretrizes/ DPOC.pdf.

9. Etemadinezhad S, Alizadeh A. Valores de referência para espirometria em adultos saudáveis na província de Mazandaran, Irã. J Bras Pneumol [Internet] 2011 Sept-Oct [acesso 2016 Nov 15]; 37(5):615-620. Disponível em: http://www. scielo.br/pdf/jbpneu/v37n5/v37n5a08.pdf. doi: http://dx.doi.org/10.1590/ S1806-37132011000500008.

10. Global Initiative for Chronic obstructive Pulmonary Disease. Global strategy for diagnosis, management, and prevention of COPD- Update 2016. Medical Commnications Resources [Internet]. 2016 [acesso 2016 Nov 15]. Disponível em: http://goldcopd.org/wp-content/uploads/2016/04/GOLD_Report_ References_2016.pdf.

11. American Thoracic Society/European Respiratory Society. Statement on respiratory muscle testing. Am J Respir Crit Care Med [Internet]. 2002 Oct [acesso 2016 Nov 15];166(4):518-624. Disponível em: http://www.atsjournals. org/doi/abs/10.1164/rccm.166.4.518. doi: 10.1164/rccm.166.4.518.

12. Corrêa KS, Karloh M, Martins LQ, Santos K, Mayer A. O teste de AVD-Glittre é capaz de diferenciar a capacidade funcional de indivíduos com DPOC da de saudáveis? Rev Bras Fisioter [Internet]. 2011 Nov-Dec [acesso 2016 Nov 15];15(6):467-473. Disponível em: http://www.scielo.br/pdf/rbfis/v15n6/pt aop033 11.pdf. doi: http://dx.doi.org/10.1590/S1413-35552011005000034.

13. Brito RR, Probst VS, Andrade AFD, Samora GAR, Hernandes NA, Marinho PEM. Reference equations for the six-minute walk distance based on a Brazilian multicenter study. Braz J Phys Ther [Internet]. 2013 Nov-Dec [acesso 2016 Nov 15];158(5):1384-1387. Disponível em: http://www.scielo.br/pdf/ rbfis/v17n6/1413-3555-rbfis-35552012005000122.pdf.. doi: 10.1590/S141335552012005000122. PubMed PMID: 24271092.

14. Azevedo KRS. Avaliação Funcional Pulmonar na DPOC. Rev Pulmão RJ
[Internet]. 2013 Mar-Apr [acesso 2016 Nov 15];22(2):24-29. Disponível em: http://www.sopterj.com.br/profissionais/_revista/2013/n_02/06.pdf.

15. Rondelli RR, Oliveira NA, Dal Corso S, Malaguti C. Uma atualização e proposta de padronização do teste de caminhada de seis minutos. Rev Fisioter Mov [Internet]. 2009 Apr-Jun [acesso 2016 Nov 15]; 22(2):249-259. Disponível em: http://www2.pucpr.br/reol/public/7/archive/0007-00002708-ART11.PDF.

16. Rodrigues SL, Viegas CAA. Estudo de correlação entre provas funcionais respiratórias e o teste de caminhada de seis minutos em pacientes portadores de doença pulmonar obstrutiva crônica. J Bras Pneumol [Internet]. 2002 NovDec [acesso 2016 Nov 15];28(6):324-328. Disponível em: http://www.scielo. br/pdf/jpneu/v28n6/a05v28n6.pdf. doi: http://dx.doi.org/10.1590/S010235862002000600005 .

17. Guyatt GH, Pugsley SO, Sullivan MJ, Thompson PJ, Berman L, Jones NL et al. Effect of encouragement on walking test performance. Thorax [Internet]. 1984 May [acesso 2016 Nov 15];39:818-822. Disponível em: http://thorax. bmj.com/content/thoraxjnl/39/11/818.full.pdf. doi: http://dx.doi.org/10.1136/ thx.39.11.818.

18. Oliveira LA, Mesquita R, Brito IL, Laburú VM, Pitta F, Probst VS. Relação do trabalho desenvolvido em testes máximo e submáximo de capacidade de exercício com o grau de obstrução ao fluxo aéreo em indivíduos com Doença Pulmonar Obstrutiva Crônica. Fisioter Pesq [Internet]. 2014 Jan-Mar [acesso 2016 Nov 15]; 21(1):81-86, 2014. Disponível em: http://www.scielo. $\mathrm{br} / \mathrm{pdf} / \mathrm{fp} / \mathrm{v} 21 \mathrm{n} 1 / \mathrm{pt}$ 1809-2950-fp-21-01-00081.pdf. doi: http://dx.doi. org/10.1590/1809-2950/484210114.

19. Carter R, Holiday DB, Nwasuruba C, Stocks J, Grothues C, Tiep B. 6-minute walk work for assessment of functional capacity in patients with COPD. CHEST [Internet]. 2003 May [acesso 2016 Nov 15];123(5):1408-1415. Disponível em: http://journal.chestnet.org/article/S0012-3692(15)33670-9/pdf. doi: http:// dx.doi.org/10.1378/chest.123.5.1408.

20. McNamara RJ, McKeough ZJ, McKenzie DK, Alison JA. Water-based exercise in COPD with physical comorbidities: a randomised controlled trial., Eur Respir J [Internet]. 2013 Jun; 41(6): 1284-1291. Diponível em: http://erj.ersjournals. com/content/erj/41/6/1284.full.pdf doi: 10.1183/09031936.00034312.

21. Bowen JB, Votto JJ, Thrall RS, Haggerty MC, Stockdale-Woolleyet R, Bandyopadhyay $\mathrm{T}$ et al. Functional status and survival following pulmonary rehabilitation. CHEST [Internet]. 2000 Sept [acesso 2016 Nov 15]; 118(3):697703. Disponível em: http://journal.chestnet.org/article/S0012-3692(15)364758/pdf. doi: http://dx.doi.org/10.1378/chest.118.3.697.

\section{Como citar este artigo/How to cite this article:}

Bastos KKRT, Olieira RCA, Lima WAM, Badaró RR, Costa KI, Couto PLS. Correlação entre capacidade funcional e capacidade pulmonar em pacientes com doença pulmonar obstrutiva crônica J Health Biol Sci. 2018 Out-Dez; 6(4):371-376. 